\title{
Efficiency Optimisation of a Forestry Crane by Implement Hydraulics with Energy Recovery
}

\author{
M. Sc. Chris Geiger, Prof. Dr.-Ing. Marcus Geimer, \\ Karlsruhe Institute of Technology, Institute of Mobile Machines, \\ Karlsruhe
}

\section{Abstract}

Forwarders are an essential part in fully mechanised timber harvesting chains. Due to a suboptimal energy usage at the implement hydraulics, caused by unused energy recovery, there is a great potential for an optimisation of the machines to increase its sustainability and environmental compatibility. Hence, innovative solutions for this challenge are designed within the project 'Forwarder2020' at the Karlsruhe Institute of Technology (KIT), embedded in and sponsored by the European program 'Horizon 2020', managed by the project leader HSM Hohenloher Spezial-Maschinenbau GmbH \& Co. KG.

The focus in this treatise is on the energy efficiency of a forestry crane, for example mounted on a forwarder. On current machines there is no built-in system to recover energy. An energy recuperation and regeneration system is therefore developed for forestry cranes.

To compare the efficiency of different machines or system architectures and to evaluate the energy recovery potential of loading processes, reference loading cycles have been established based on field measurements of real logging processes. These standardized reference cycles represent recurrent loading cycles in a working environment.

\section{Motivation}

In a fully mechanised harvesting process like cut-to-length (CTL) logging system, a harvester fells the trees and crosscuts them into specific lengths. This pre-processing includes a separation of the logs into different assortments with characteristic lengths. In the next process step forwarders are loading the logs and move them from the felling site to forestry roads. There, the forwarder unloads the logs and piles them to specific assortments, whereby a further transportation via forestry trucks is easily achievable. [1][2]

In Nordic countries like Sweden or Finland, up to $100 \%$ of the harvest is achieved by the CTL-system, whereas in Germany it is used about $35 \%$. As forwarders are used for the logging process in motor manual systems too, they are an essential part in mechanised timber harvesting chains. [3] 
The main task of a forwarder is loading and unloading of logs, which sums up to $80-85 \%$ of total working hours, by what means $15-20 \%$ of the time forwarders are driving [4]. Regarding to this, loading processes are an important starting point for an energy efficiency improvement. During these loading cycles, there are common situations like lowering the crane or a practically horizontal movement of the grapple where the potential energy of the system due to active loads can be reused.

Hence, in the first part of this paper, loading cycles of forwarders used in CTL systems are examined to derive characteristic patterns for further evaluations and to estimate the energy recovery potential of forestry cranes based on this reference cycles.

As there are no built-in systems for energy recovery on in-market machines, the second part of this paper describes an innovative system for energy recuperation and regeneration in crane systems of forwarders. The efficiency improvement potential will exemplary be shown on the defined loading cycles. With these reference cycles, different machines and systems can be compared in relation to energy efficiency.

\section{Loading cycles of forwarders used in cut-to-length systems}

To achieve a sufficient database for the evaluation of loading cycles, measurements in the forest under real conditions in a CTL thinning process took place. Therefore, an in-market machine with a crane as shown in figure 1 was used. The crane consists of a crane pillar, inner boom, outer boom and telescope including the related cylinders. The forwarder was equipped with sensors for cylinder positions and chamber pressures for all consumers. The pressure and flow supplied by the hydraulic pump were also measured. Furthermore, the control signals from the crane joysticks were recorded as well as significant engine variables like engine speed or fuel consumption. With these data, the energy consumption of the machine or individual consumers can be determined, as well as characteristic movement patterns for loading processes, resulting in reference loading cycles.

Figure 2 shows exemplarily for a forest alley the determined distribution of the collected logs, whereas the centre of the crane pillar is located at $x=y=0 \mathrm{~m}$. The distribution shows clearly the machine operators' preferred position of the forwarder for the loading process, which is rectangular towards the log at the height of the crane pillar. At this position, the view over the machine, logs to be picked up and environment enables the machine operator to execute an optimal loading process. This results corresponds to similar experiments carried out in [5].

Based on observations of forwarders working in forest alleys, a loading cycle of a forwarder includes the following phases: 
I. Motion of the grapple from the load bunk to the log

II. Gripping of the log

III. Retraction motion of the grapple including the log towards the load bunk

IV. Positioning and dropping of the log
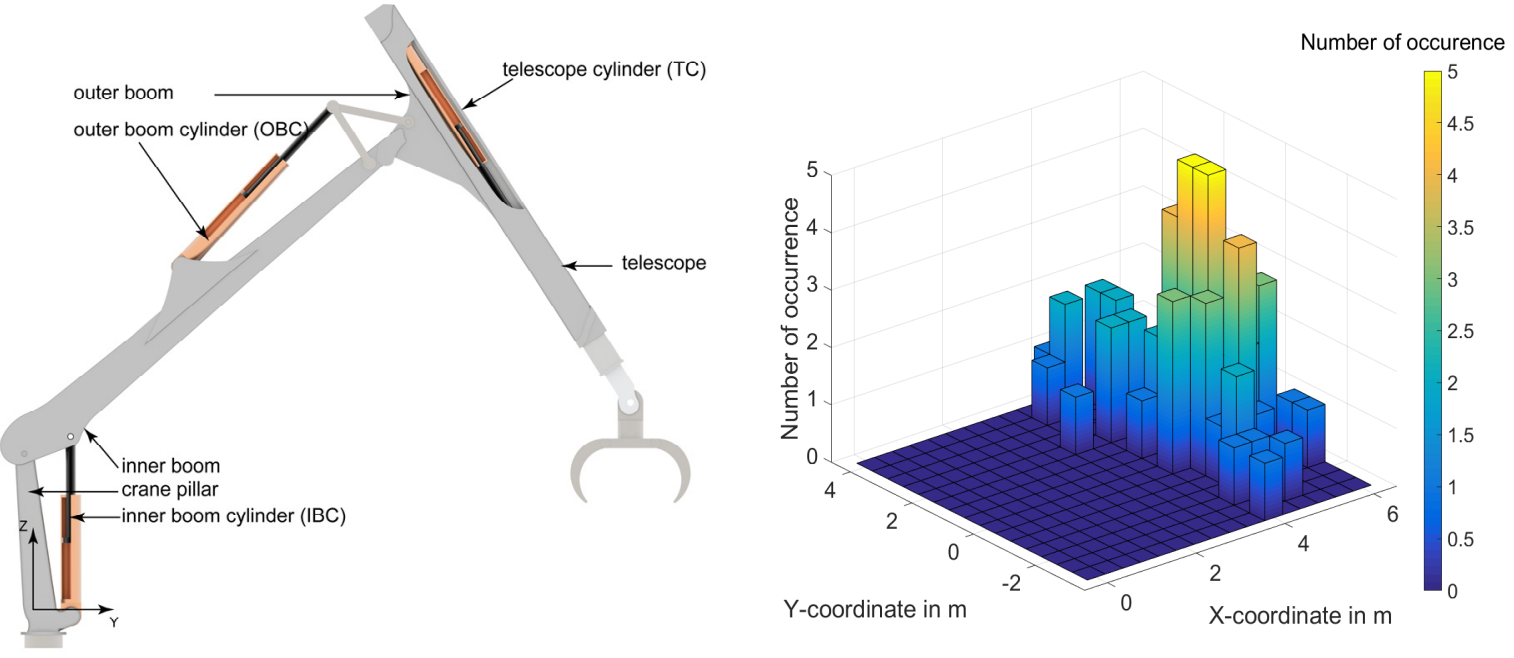

Fig. 1: Schematic sketch of a forestry

Fig. 2:Spatial distribution of collected logs crane

For a first evaluation of the loading cycles, the position of the inner boom cylinder (IBC), cf. figure 1 , is compared for different loading cycles measured in the forest. Figure 3 shows the normalized position of the IBC for randomly chosen loading processes measured in a forest alley. Hereafter, all cylinders are fully extracted at position 1. For a comparison of loading cycles the IBC is more suitable as loading cycles start and end with a movement of the IBC than one of the other cylinders, cf. figure 3. Remarkable is that the characteristic profile of each loading process seams similar, although the starting position and loading time differs. 
The difference in the starting position is caused by an increasing loading height due to more logs in the load bank. The time difference, resulting in more dispersed lines starting at $\mathrm{t}=10 \mathrm{~s}$, occurs because of varying positions of the logs, the difficulty of gripping logs lying on the ground or different retracting movements towards the load bank due to manoeuvring around not chopped trees. The exact movement pattern is explained in chapter

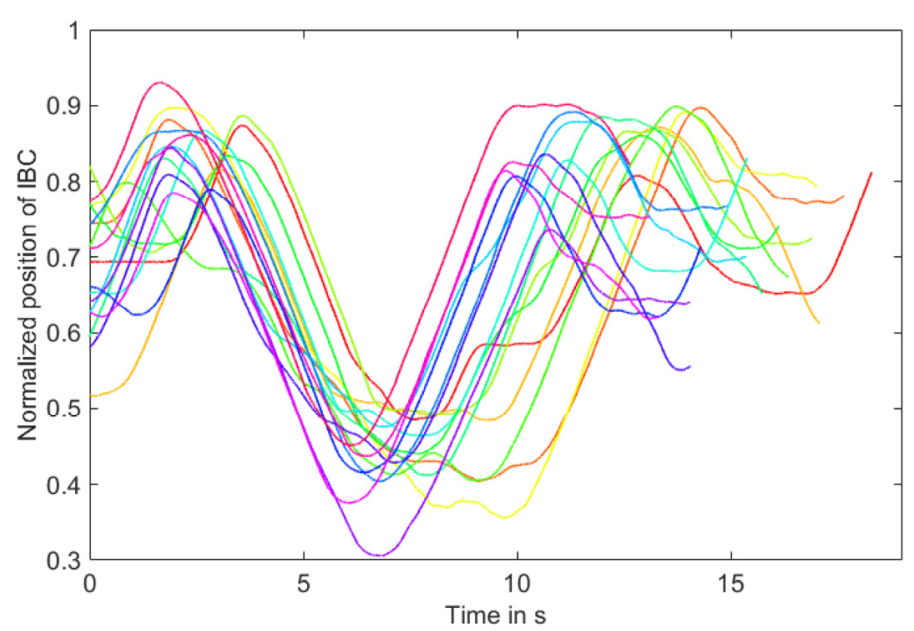

Fig. 3: Normalized position of inner boom cylinder for different loading cycles

3.

Combining the results shown in figure 2 and figure 3 , machine operators prefer a specific relative position of a forwarder towards the logs and following a characteristic movement pattern to load the log. These conclusions are in accordance with results achieved in [1] and [5]. To map these findings onto reproducible reference cycles, a laboratory test setup as shown below was developed and installed following [6]. Thus, deviations in the cylinder position at the beginning of the cycle or varying loading times can be prevented. With this reference cycles efficiency of different machines or system architectures can be compared.

\section{Definition of normalized loading cycles}

The laboratory test setup is shown in figure 4. Corresponding to the achieved results, a log (length $4 \mathrm{~m}$, weight $320 \mathrm{~kg}$ ) is placed at a log centre distance of $4.6 \mathrm{~m}$ and $8.5 \mathrm{~m}$ next to the machine to cover significant, realistic log positions. $8.5 \mathrm{~m}$ are considered because in the future field of application, the number of forest alleys should be decreased to reduce the impact of forestry machines on the soil, whereby environmental sustainability is enhanced. By following the findings displayed in figure 2 , the position in y-direction of the log is chosen between crane pillar and first tyre of the front wagon. The load bunk of the forwarder is empty. Several operators repeated the loading process for each distance at least 15 times. The measurement for each loading cycle starts with the crane tip placed centred over the empty load bunk and stops when the same position is achieved after putting the log in the load bunk. During the loading cycle, the movement follows the steps as described in chapter 2. 


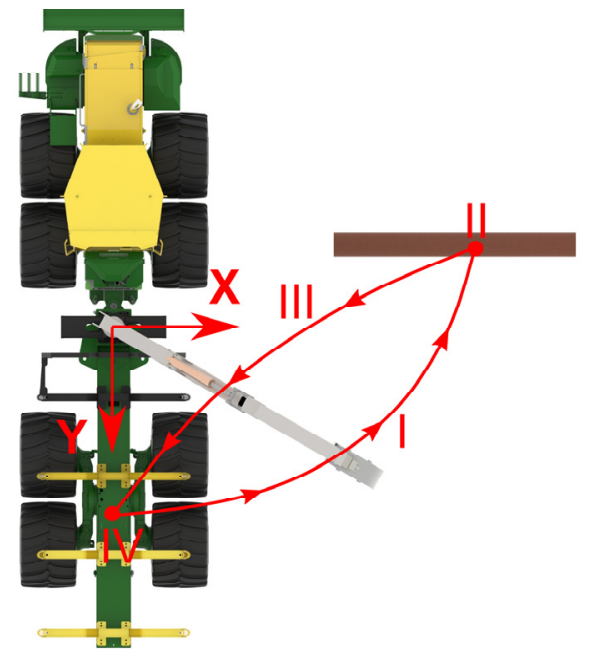

Fig. 4: Laboratory test setup

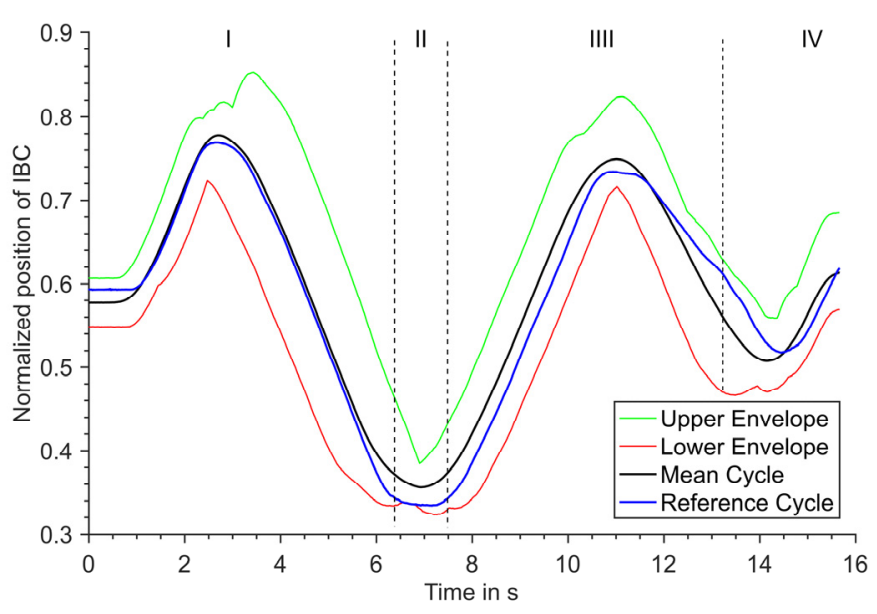

Fig. 5. Normalized position of inner boom cylinder

Exemplarily for middle-distance ranges, the total loading cycle times differ about $7 \%$ around a mean value of $15.2 \mathrm{~s}$. To compare the different cycles and filter out reference cycles which fits best a mean cycle, the time series of the loading cycles are scaled to an equal length. This principle is widely used and known as Uniform Scaling (US) and described in different treatises [6][7]. After using the principle of US, a mean loading cycle for middle-distance ranges is achieved by taking the arithmetic mean of all cycles.

The black graph in figure 5 shows exemplarily the mean position of the IBC over time. The green respectively red graph represents the upper respectively lower envelope of the measured loading cycles. The loading process is divided into four phases as characterised in chapter 2. Striking is the small deviation of the amplitude and loading time of the considered cycles, which confirms that the operators are highly skilled and experienced.

By calculating the normalized root mean square error (NRMSE), the alignment quality between a tested cycle and the mean cycle can be compared. The reference loading cycle is equivalent to the cycle with the lowest NRSME. This reference cycle for middle-distance ranges (log centre distance of $4.6 \mathrm{~m}$ ) is shown in figure 5 via the blue graph. Same proceeding is done with the data series of the other consumers, e.g. the OBC, leading to the same loading cycle as reference cycle.

By using US and NRSME, an independent reference cycle can be evaluated. This procedure can be repeated for the significant log positions, which concludes in a reference cycle for both middle-distance and long-distance ranges. 


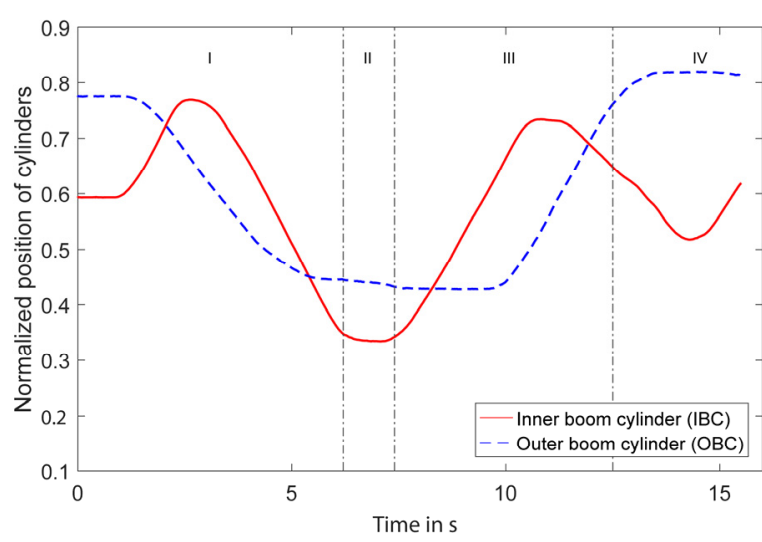

Fig. 6: Reference cycle for middle-distance ranges

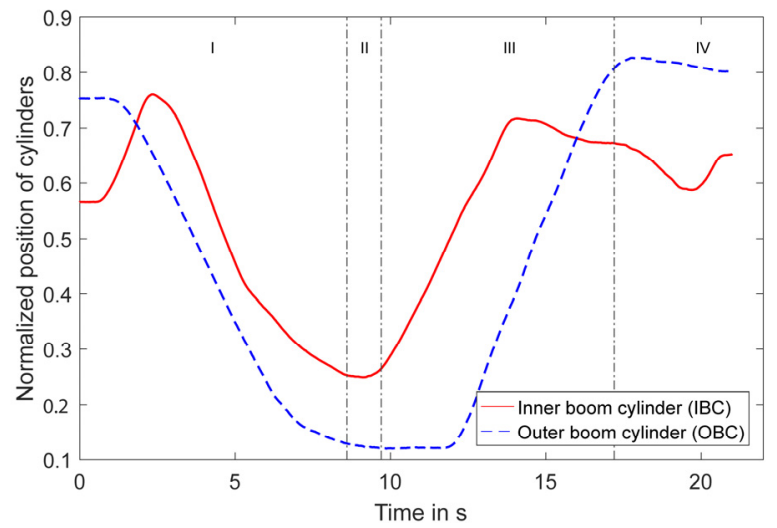

Fig. 7: Reference cycle for long-distance ranges

The course of both IBC and $\mathrm{OBC}$ of the reference cycle for middle-distance ranges is displayed in figure 7, for long-distance ranges in figure 8 . Although they show different cycle times, the characteristic movement pattern is similar. At the beginning, the crane tip has to be lifted over the load bunk poles, resulting in an extension of the IBC. Simultaneously, the $\mathrm{OBC}$ is retracted and therefore the crane tip moving lateral from the crane pillar. After passing the poles, the grapple is moved directly towards the log by lowering the inner boom and lifting the outer boom, whereby the telescope can be extracted (not illustrated). At the lowest position of IBC and $\mathrm{OBC}$, the log is picked up. Afterwards it is retracted towards the forwarder while at the same time lifted over the poles (IBC and OBC extending) and finally lowered down into the empty load bunk (IBC retracting). The inner boom upward movement at the very end of the cycle results from positioning the crane tip at the starting point.

With these achieved reference cycles for both middle-distance and long-distance ranges, a reproducible evaluation of energy efficiency of different machines and system architectures is possible. Furthermore, the theoretical potential for energy recuperation can be estimated.

\section{Energy recovery potential of forestry cranes of forwarders}

As seen in figures 6 and 7, there are frequent situations where the inner boom cylinder (IBC) and outer boom cylinder (OBC) are acting simultaneously. As a result, exemplary for a lateral movement of the crane tip, the inner boom is lowered while the outer boom has to be lifted at the same time. Therefore, theoretically all of the potential energy from lowering the inner boom could be transferred to the outer boom, which would make the motion way more energy efficient. Energetically this means an idealized horizontal, lateral movement of the crane tip neglecting friction and further losses does not require any energy. 
Figure 8 respectively figure 9 shows the position of IBC (red graph) and OBC (blue dashed graph) over cycle time and the theoretical usable power due to lowering inner or outer boom for middle-distance ranges respectively long-distance ranges. This power is calculated for time ranges when $\mathrm{IBC}$ is retracting and $\mathrm{OBC}$ extending, as only there energy reuse is possible, by equation 1 :

$$
P=\Delta p \cdot Q
$$

with $P$ : Power in $\mathrm{W}$

$\Delta \mathrm{p}: \quad$ Pressure difference between cylinder and environment in $\mathrm{Pa}$

Q: $\quad$ Volume flow in $\mathrm{m}^{3} / \mathrm{s}$

Most potential for energy reuse is in the IBC taking into account that the total mass of the crane is lowered. As the mass of the outer boom is significant lower, also the usable power is less than for the inner boom while lowering down. Furthermore, the time proportion is less than for the IBC.

There are significant periods where both $\mathrm{IBC}$ and $\mathrm{OBC}$ are extending or retracting. Here, an energy regeneration is possible by transferring the usable power between the different cylinders. For example, taking the reference cycle as shown in figure 8 , from $2.5 \mathrm{~s}$ on the IBC is retracting while the $\mathrm{OBC}$ is expanding.
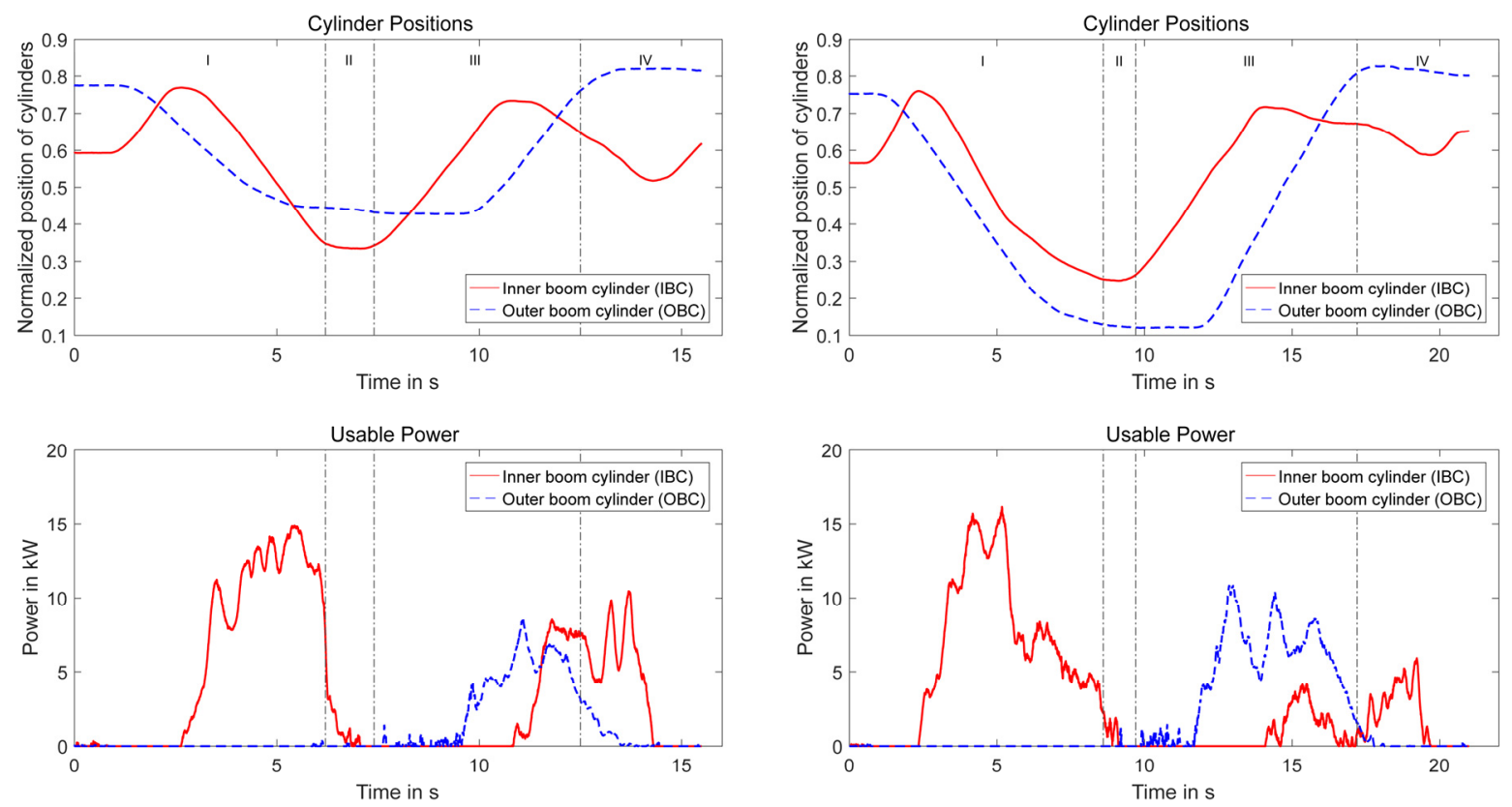

Fig. 8: Middle-distance reference cycle

Fig. 9: Long-distance reference cycle

The released power from the IBC can be transferred to the $\mathrm{OBC}$ and be used for the process of lifting the outer boom. Same proceeding can be used inversely from $10 \mathrm{~s}$ on to support the 
lifting of the inner boom. Another possibility is to recuperate the released energy, e.g. by using a hydraulic accumulator. In this case, the energy released during lowering processes is stored temporarily and reused e.g. for lifting processes.

In comparison, the reference cycle pictured in figure 9 for long-distance ranges shows a similar pattern. Here the periods where energy regeneration is possible are longer compared to the cycle shown in figure 8. As the log is arranged further away from the machine, the inner boom has to be lowered more than in middle distances, simultaneously the outer boom has to be lifted more during extending, cf. the time period up to $9 \mathrm{~s}$ in figure 9 .

\section{Concept of energy-saving crane system}

The concept of an energy-saving crane system is shown in figure 10 [8]. With this hydraulic system, both

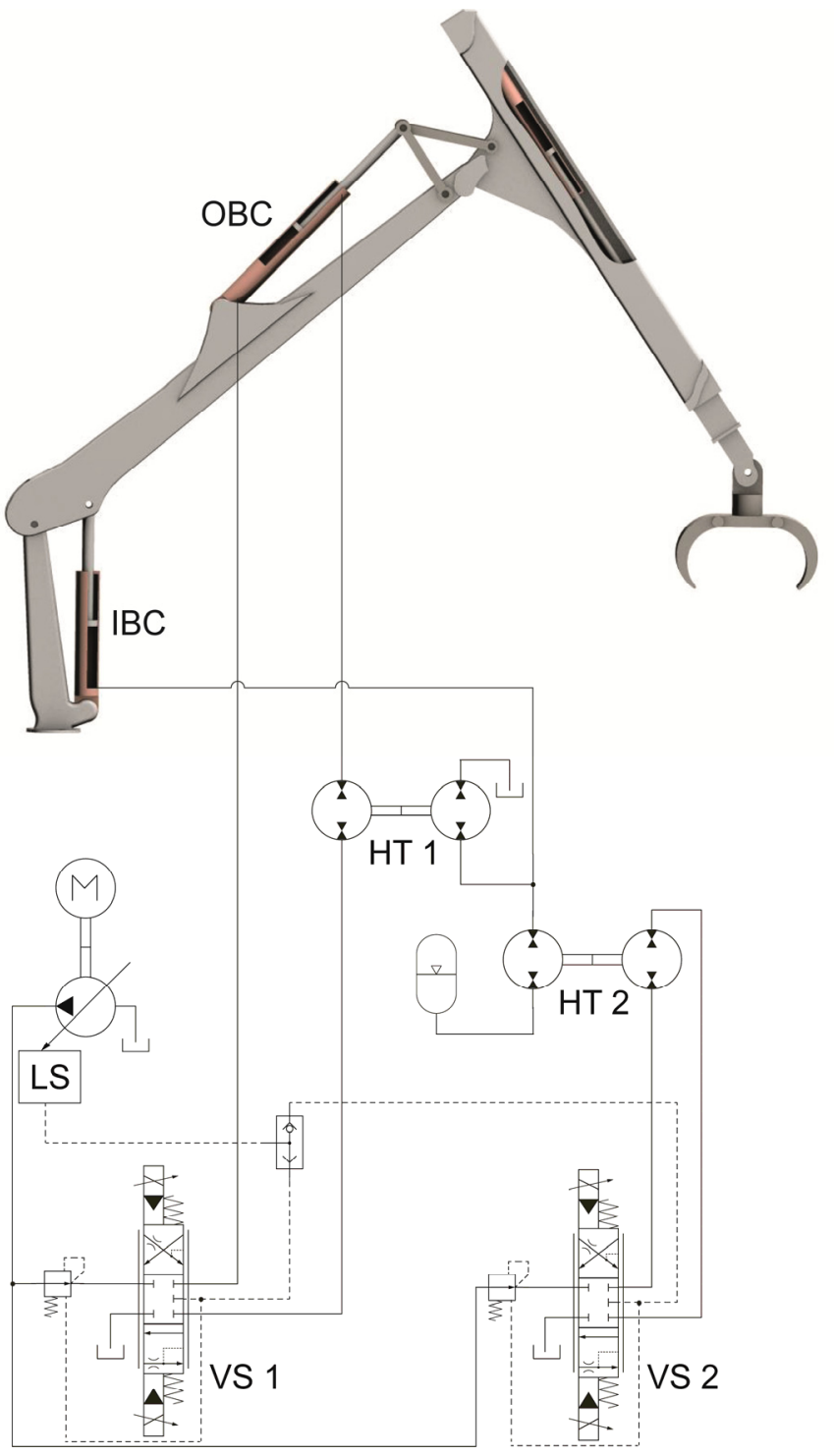

Fig. 10: Circuit diagram of new hydraulic system [8] energy recuperation and regeneration are possible. This system can be used independently from specific crane designs as the interfaces are between the crane and the valve block mounted on the forwarder. The system connects the IBC and OBC by a hydraulic transformer. The hydraulic transformer converts the usable power $\mathrm{P}$ by adjusting the pressure difference and volume flow, cf. equation 1, for the different cylinders.

For a further explanation of the innovative system, an extending, lateral and horizontal movement serves as a simplified example. This motion implies lowering the inner boom while simultaneously lifting the outer boom. For this, valve section (VS) VS 1 is actuated while VS 2 remains in normal position, so that there is no volume flow over the hydraulic trans- 
former HT 2. Therefore, oil flows from the bottom chamber of IBC towards the hydraulic transformer HT 1, resulting in a movement of the HT 1 as VS 1 is additionally actuated. This leads to a volume flow from the left side of HT 1 towards OBC, while simultaneously the emitted power from the right side is transferred via a mechanical connection to the left side. Due to this, the hydraulic pump needs to supply the same volume flow, but with a significant lesser oil pressure compared to a state-of-the-art system. In this modus energy regeneration takes place.

If additionally the crane tip has to be lowered down, either valve section VS 2 has to be switched or the hydraulic transformer has to be manipulated in a way that the left and right side are mechanically decoupled. By that, oil flows through the hydraulic transformer HT 2 into the accumulator and is recuperated. By switching VS 2 into the opposite position, the accumulated oil flows towards the IBC resulting in lifting the inner boom. Especially considering several loading processes, the stored energy of lowering the grapple including logs towards the load bunk (cf. phase four) can be used to lift the crane over the load bunk poles for the next loading process, cf. figures 8 and 9.

\section{Conclusion and Perspectives}

Reference loading cycles for middle- and long-distances ranges for forestry cranes used in CTL-processes are determined via measurements under real working conditions. Based on these reference cycles, the energy recovery potentials of cranes mounted on a forwarder are evaluated. Furthermore, an innovative hydraulic system for energy regeneration and recuperation including hydraulic transformers is presented, which will reduce the fuel consumption of forwarders significantly as the motor load decreases.

In further steps, a simulation model of a forestry crane, which combines a multi-body and a hydraulic simulation, integrated in a holistic model of a forwarder, can be parameterized and validated. The energy efficiency of the innovative hydraulic concept can be determined by modifying the validated simulation model, including a parameter study for an optimal configuration of the integrated system. The reference cycles will be used to compare the energy efficiency of the energy-saving crane system to current state of art. Afterwards, a demonstrator including the new hydraulic system will be set up and measured. 


\section{Acknowledgements}

This project has received funding from the European Union's Horizon 2020 research and innovation programme under grant agreement No 727883 .

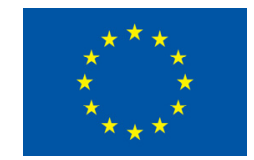

\section{References}

[1] Morales, D.: Increasing the Level of Automation in the Forestry Logging Process with Crane Trajectory Planning and Control. Journal of Field Robotics 31(3), p. 343-363, 2014

[2] Manner, J.: Automatic and Experimental Methods to Studying Forwarding Work. PhD thesis, Swedish University of Agricultural Sciences, 2015

[3] Cacot, E.: Developing full-mechanized harvesting systems for broadleaved trees: a challenge to face the reduction of the manual workforce and to sustain the supply of hardwood industries. 2006 Council on Forest Engineering (COFE) Conference Proceedings: "Working Globally - Sharing Forest Engineering Challenges and Technologies Around the World" Coeur d'Alene, July 22-Aug 2,2006

[4] Manner, J.: Load level forwarding work element analysis based on automatic follow-up data. Silva Fennica vol. 50 no. 3, 2016

[5] Morales, D.: Path-Constrained Motion Analysis: An Algorithm to Understand Human Performance on Hydraulic Manipulators. IEEE TRANSACTIONS ON HUMANMACHINE SYSTEMS, VOL. 45, NO. 2, 2015

[6] Deiters, H.: Standardisierung von Lastzyklen zur Beurteilung der Effizienz mobiler Arbeitsmaschinen. PhD thesis, Technische Universität Braunschweig, 2009

[7] Yankov, D.: Detecting Time Series Motifs Under Uniform Scaling. Proceedings of the 13th ACM SIGKDD international conference on Knowledge discovery and data mining, San Jose, California, USA, 2007

[8] Hohenloher Spezial-Maschinenbau GmbH \& Co. KG. Gebrauchsmusterschrift DE 20 2014006861 U1, 2015 